IS THERE A RELATIONSHIP BETWEEN THE T PEAK TO END INTERVAL AND RIGHT BUNDLE BRANCH BLOCK?

\author{
A. Benatar ${ }^{1}$, T. Decraene ${ }^{2}$, A. Feenstra ${ }^{1}$ \\ ${ }^{1}$ Paediatric Cardiology, ${ }^{2}$ Universitair \\ Kinderziekenhuis Brussel, Brussels, Belgium
}

Background: The interval between the peak and end of the T wave (TpTe) is an index of spatial dispersion in transmural cardiac repolarization. We set out to examine the behaviour of TpTe in the presence of right bundle branch block (RBBB) as a potential marker for arrhythmogenesis.

Methods: 42 children with RBBB were enrolled. In 39 RBBB occurred post cardiac surgery, in 3 secondary following cardiomyopathy. From a resting 12-lead digital ECG, TpTe, RR, QT, and JT intervals were measured (leads II and V5). Bazett and Fridericia formulas were applied to TpTe for heart rate correction and TpTe/QT and TpTe/JT were calculated. Results were compared to normal data (400 children). Descriptive and analytical statistics significance level set at $p<0.05$.

Results: Median age 7,7 years $\pm 5,9$. Mean QRS duration 120ms (range 88-168 ms). TpTe lead II 93 $\mathrm{ms} \pm 15$, V5 $95 \mathrm{~ms}+14$, lead II TpTeB $118 \pm 23$, TpTeF $109+\underline{19}$, V5 TpTeB $120 \pm 20 ;$ TpTeF 111 + 17. TpTe/JT II 0,42 + 0,1 and 0,42+0,1, TpTe/ QT II 0,27+0.04 and TpTe/QT V5 0,27+0,04. All the values measured were significantly different $p<$ 0.05 from control data.

Conclusion: TpTe reflects global myocardial repolarization and is a surrogate diagnostic parameter. In our cohort of RBBB patients, TpTe and ratio to JT and QT were found to be prolonged. No patients to date have developed important ventricular arrhythmia. The role of right ventricular size and haemodynamics on TpTe is under investigation.

\section{DOPPLER-DERIVED DIASTOLIC VENTRICULAR FUNCTION IN INFANTS WITH BIRTH WEIGHT LESS THAN 1250 G DURING FIRST 48 HOURS OF LIFE}

\author{
J. Sirc ${ }^{1,2}$, E.M. Dempsey ${ }^{3,4}$, J. Miletin ${ }^{1,2}$
}

${ }^{1}$ Department of Paediatric and Newborn Medicine, Coombe Women and Infants University Hospital,

Dublin, Ireland, ${ }^{2}$ Neonatal Intensive Care Unit, Institute for the Care of Mother and Child, Prague,

Czech Republic, ${ }^{3}$ Department of Neonatology, Cork University Maternity Hospital, ${ }^{4}$ Department of Paediatrics and Child Health, University College Cork, Cork, Ireland

Background and aims: To assess diastolic function in preterm infants less than $1250 \mathrm{~g}$ during first 48 hours of life and correlate with clinical parameters and cardiac output measurements.

Methods: A prospective observational study. Newborns with birth weight $<1250 \mathrm{~g}$ were eligible for enrolment. Newborns with congenital heart disease (excluding patent ductus arteriosus) or major congenital malformations were excluded. Doppler-derived parameters (early and atrial filling time velocity integral, early filling acceleration time, atrial filling fraction and isovolumic relaxation time) were measured on tricuspid and mitral valves at 6 , 12, 24 and 48 hours of age. Ductal patency and flow was also assessed and superior vena cava flow was measured.

Results: 10 neonates were enrolled following parental consent. The mean birth weight was 767 g (SD \pm 184$)$, mean gestational age was 25.3 weeks (SD \pm 1.4$)$. The mean tricuspid valve isovolumic relaxation time (TV IVRT) at 6 hrs of age was 76 $\mathrm{ms}$, at 12 hours $74 \mathrm{~ms}$, at 24 hours $60 \mathrm{~ms}$ and at 48 hours $60 \mathrm{~ms}$. The mean mitral valve isovolumic relaxation time (MV IVRT) at 6 hrs of age was 58 $\mathrm{ms}$, at 12 hours $57 \mathrm{~ms}$, at 24 hours $47 \mathrm{~ms}$ and at 48 hours $52 \mathrm{~ms}$. There was a statistically significant negative correlation between MV IVRT and superior vena cava flow (SVC) $(r=-0.45, p=0.004)$.

Conclusion: This is the first description of Dopplerderived diastolic ventricular function parameters in extremely preterm infants during first 48 hours of life. 